\title{
HIGH-SPEED MONITORING OF DUST PARTICLES IN ITER ELMs SIMULATION EXPERIMENTS WITH QSPA KH-50
}

\author{
Vadym A. Makhlaj ${ }^{a, *}$, Igor E. Garkusha ${ }^{a}$, Nikolay N. Aksenov ${ }^{a}$, \\ Alexander A. Chuvilo ${ }^{a}$, Igor S. LANDman ${ }^{b}$ \\ ${ }^{a}$ Institute of Plasma Physics of the NSC KIPT, 61108 Kharkov, Ukraine \\ ${ }^{b}$ Karlsruhe Institute of Technology (KIT), IHM, 76344 Karlsruhe, Germany \\ * corresponding author: makhlay@kipt.kharkov.ua
}

\begin{abstract}
Dust generation under powerful plasma stream impacts has been studied in ITER ELM simulation experiments with QSPA Kh-50 plasma accelerator. Repetitive plasma exposures of tungsten have been performed by $0.25 \mathrm{~ms}$ plasma pulses and the heat load varied in the range $(0.1 \div 1.1) \mathrm{MJ} \mathrm{m}^{-2}$. Main characteristics of dust particles such as a number of ejected particles, their velocity, angular distribution and start time from the surface are investigated. Dust particles have not been observed under heat load below the cracking threshold. Quantity of dust particles rises with increasing heat load. Average velocities of dust particles are found to be strongly dependent on their start time from the surface after beginning of plasma-surface interaction. Maximal velocity achieved a few tens of meters per second.
\end{abstract}

KEYWORDS: ITER, ELMs simulation experiments, QSPA, plasma-surface interaction, tungsten dust.

\section{INTRODUCTION}

The anticipated regime of the tokamak ITER is the ELMy H-mode. The edge localized modes (ELMs) of plasma instabilities, intrinsic for H-mode, produce short periodic pulses of heat flux at the divertor armor. Tungsten is the most suitable material for the ITER divertor. It should withstand both the stationary and transient heat fluxes demonstrating tolerably erosion rate. The drawbacks of tungsten as plasma-facing material are $\mathrm{W}$ brittleness and damage effects related with brittle destruction as well as the melt layer erosion 11. Tungsten cracking leads to generation of the dust, which can contaminate and radiatively cool the core plasma [7, 4. The dust particles produced during tungsten cracking and the droplets ejected from the melt layer under the action of giant ELMs and disruptions are critical issues for ITER performance that require comprehensive experimental studies.

There are three main approaches to experimental dust study in fusion plasmas: (i) collection of dust particles resulted from transient events, (ii) laser scattering by the dust grain sand and (iii) dust monitoring with fast cameras 6. Observations with fast cameras can track the trajectories of the grains in the chamber during the discharge (with trajectory reconstruction), giving the magnitude of dust speed. It can also provide information on grain-wall collisions, some peculiar features of dust dynamics in fusion reactor, the amount of visible dust grains in vacuum chamber during operation in different discharges and regimes [6].

The ITER energy fluxes to the divertor are not achievable in existing tokamaks. For this reason simulation experiments of ITER transients have been carried out with other plasma devices [7, 4, 5].
In particular, the quasi-stationary plasma accelerators (QSPA) can reproduce energy densities $(0.2 \div$ $\left.2 \mathrm{MJ} \mathrm{m}^{-2}\right)$ and pulse duration $(0.1 \div 0.5 \mathrm{~ms})$ of ITER ELMs [7, 4. Therefore, QSPA can be applied for investigation of material response to the expected heat loads 4, 5, 3. This paper presents the results of QSPA Kh-50 experiments on high-power interactions with material surfaces and behavior of tungsten dust under pulsed energy loads typical for ITER Type I ELMs.

\section{ExPERIMENTAL SETUP}

The quasi-steady-state plasma accelerator QSPA $\mathrm{Kh}-50$ is the largest and most powerful device of this kind 44, 12. QSPA Kh-50 consists of two stages. The first one is used for plasma production and preacceleration. The main second stage is a coaxial system of shaped active electrodes with magnetically screened elements, supplied from independent power sources. The hydrogen plasma streams, generated by QSPA Kh-50, are injected into the magnetic system of $1.6 \mathrm{~m}$ in length and $0.44 \mathrm{~m}$ in inner diameter with a magnetic field of up to $0.54 \mathrm{~T}$ in diagnostic chamber $(2.3 \mathrm{~m}$ from acceleretor) where the target has been installed [3, 12. Plasma parameters were varied both by changing dynamics and the amount of gas filled in the accelerator channel and by the variation of the working voltage of capacitor battery of the accelerating channel. The main parameters of QSPA plasma streams were as follows: ion impact energy about $0.4 \div 0.6 \mathrm{keV}$, the maximum plasma pressure $3.2 \mathrm{bar}$, and the plasma stream diameter about $18 \mathrm{~cm}$. The surface energy loads measured with a thermocouple calorimeters were varied in the range 


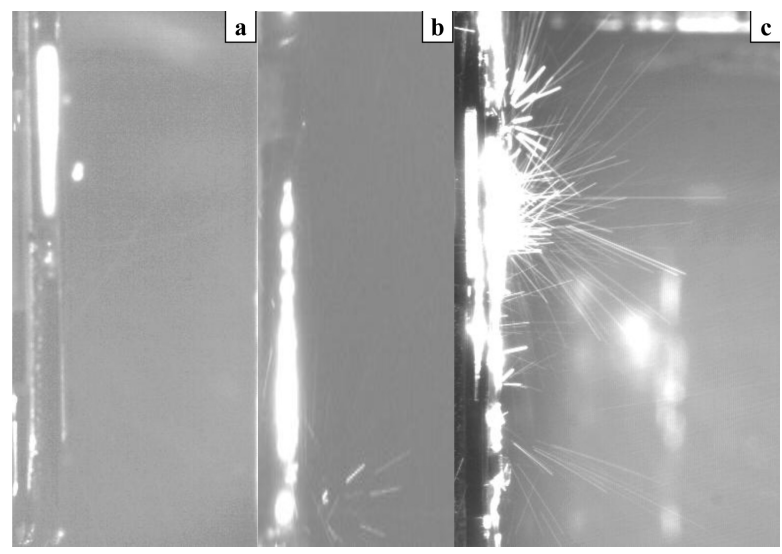

FiguRE 1. High speed imaging of plasma interaction with tungsten target: $t=1.2 \mathrm{~ms}$ after the start of plasma-surface interaction, $t_{\exp }=1.2 \mathrm{~ms}$;

a) $Q_{\text {surf }}=0.45 \mathrm{MJ} \mathrm{m}^{-2}$, b) $Q_{\text {surf }}=0.6 \mathrm{MJ} \mathrm{m}^{-2}$,

c) $Q_{\text {surf }}=0.75 \mathrm{MJ} \mathrm{m}^{-2}$.

of $(0.1 \div 1.1) \mathrm{MJm}^{-2}$ [3]. The plasma pulse shape was approximately triangular, and the pulse duration was about $0.25 \mathrm{~ms}$.

In previous experiments it was demonstrated that tungsten cracking and melting thresholds under QSPA Kh-50 exposures corresponded to $0.3 \mathrm{MJ} \mathrm{m}^{-2}$ and $0.6 \mathrm{MJ} \mathrm{m}^{-2}$ respectively. The evaporation onset is estimated as $1.1 \mathrm{MJ} \mathrm{m}^{-2}[4,3]$.

The targets made of pure tungsten of sizes $5 \times$ $5 \times 0.5 \mathrm{~cm}^{3}$ and $12 \times 8 \times 0.1 \mathrm{~cm}^{3}$ have been used for these experiments. Observations of plasma interactions with exposed surfaces, the dust particle dynamics and the droplets monitoring have been performed with a high-speed 10 bit CMOS pco.1200s digital camera PCO AG (exposure time from $1 \mu \mathrm{s}$ to $1 \mathrm{~s}$, spectral range from 290 to $1100 \mathrm{~nm}$ ).

In general, the applied measurement scheme for droplets monitoring was similar to one used in [4, 5, 3]. As an example, Fig. 1 shows camera frames registered with the same exposure for different plasma heat loads. Camera frames corresponding to different time moments during one plasma pulse are presented in Fig. 2.

Dynamics of particles ejected from exposed surfaces has been analyzed from the series of camera frames. This is the main difference of applied scheme from the experiments described in [5]. Velocities of ejected tungsten dust particles/droplets have been evaluated from the lengths of their traces done coluring selected frame. The moment when the particle was released from the target surface could be calculated also. Temporal distributions of quantity and velocity of erosion products were obtained for different heat loads to the exposed tungsten surfaces.

The observed region in front of target was $8 \mathrm{~cm}$ due to feature of a design the diagnostic vacuum chamber of QSPA Kh-50. Therefore, the particles with velocity higher than $35 \mathrm{~m} / \mathrm{s}$ were able to fly away of observed region during of $2.2 \mathrm{~ms}$. Taking into account

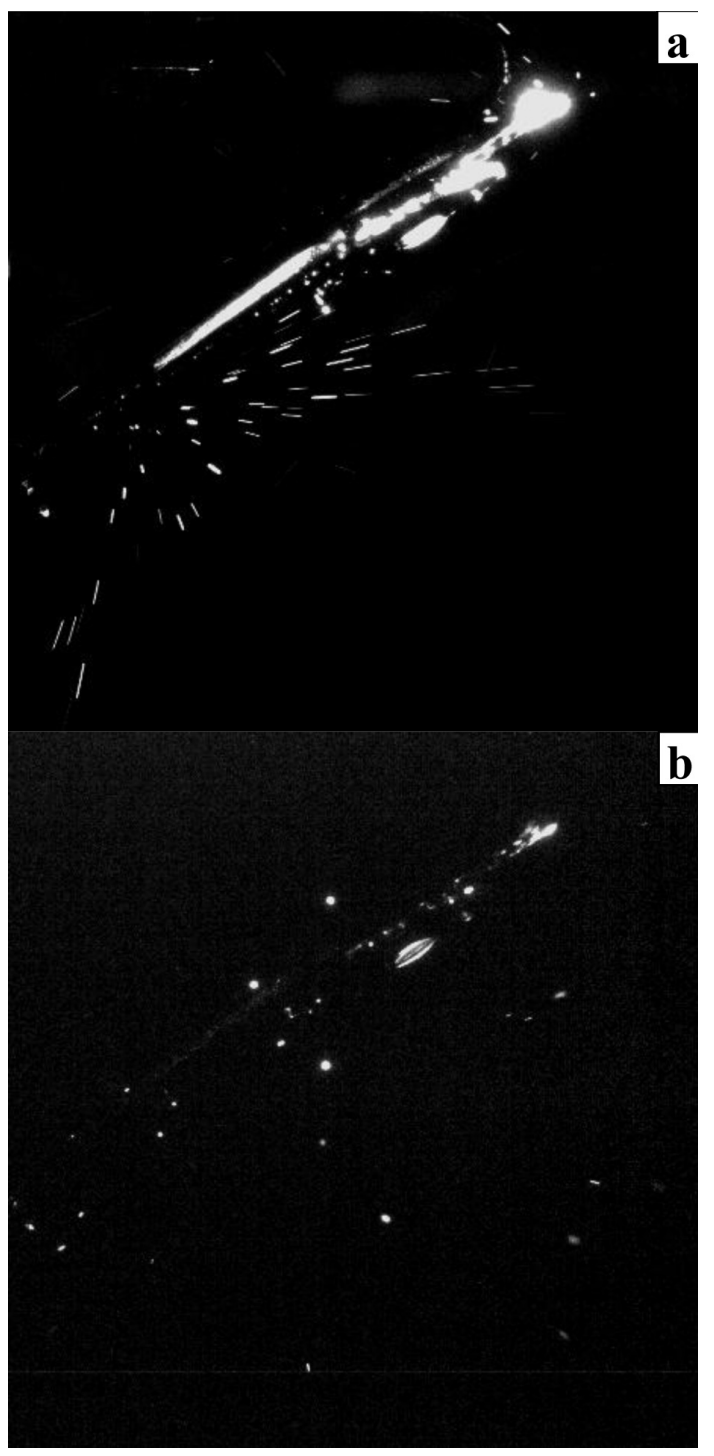

FigURE 2. High speed images of plasma interaction with inclined ( $35^{\circ}$ to plasma jet) tungsten target, a) $t=1.16 \mathrm{~ms}$ and b) $t=9.06 \mathrm{~ms}$ after the start of plasma-surface interaction, $Q_{\text {surf }}=0.75 \mathrm{MJ} \mathrm{m}^{-2}$, $t_{\exp }=0.25 \mathrm{~ms}$.

these circumstance, the special pulsed system was used for synchronization of the camera registration start and the beginning the plasma-surface interaction. This allows improvement of temporal resolution for applied registration system in comparison with that described in 5]. The exposure was not exceed $1.5 \mathrm{~ms}$. Particles were also registered after plasma impact for more than $9 \mathrm{~ms}$ (Fig. $2 \mathrm{~b}$ ). The total recording time achieved $50 \mathrm{~ms}$.

\section{EXPERIMENTAL RESUlTS}

The irradiation of tungsten surface with QSPA Kh-50 plasma heat load below the cracking threshold $\left(0.3 \mathrm{MJ} \mathrm{m}^{-2}\right)$ does not trigger the generation of erosion products. At the heat load above the cracking threshold but below melting threshold only several dust particles traces have been registered (Fig. 1p). 
Elastic energy stored in stressed tungsten surface layer should be the motive force for the cracking process with following acceleration of separated solid particles in this case 10.

Further increase of heat load leads to the surface melting and results in splashing of eroded material (Fig. 1b). Number of ejected particles rises with increasing heat load due to growing thickness of melted layer. Quantity of particles ejected from irradiated surface increases more than twice as a consequence of heat load elevation from $0.6 \mathrm{MJ} \mathrm{m}^{-2}$ to $0.75 \mathrm{MJ} \mathrm{m}^{-2}$ only. The majority of $\mathrm{W}$ particles are ejected from the exposed surface $0.2 \mathrm{~ms}$ after beginning of plasmasurface interaction (Fig. 3).

Velocity of registered tungsten particles achieves $25 \mathrm{~m} / \mathrm{s}$ for earlier instants. For the later moments, velocity decreases to several m/s [3, 9]. Maximal velocity only weakly depends on the heat load. The maximum of particles with high velocities (i.e. ejected from surface before $t=0.4 \mathrm{~ms}$ ) is clearly detected on camera frame at $1.2 \mathrm{~ms}$ after beginning of plasma-surface interaction (Fig. 3). For the later moments of observation [9], they leave a zone of observation. Therefore, the number of such particles decreases. The quantity of particles with velocity of $10 \mathrm{~m} / \mathrm{s}$ changes weakly during $5 \mathrm{~ms}$.

As follows from [3, under perpendicular plasma impacts droplets are ejected primarily with small angles to the normal (i.e. towards impact plasma, see Fig. 1. Nevertheless, rather large angles of ejection, up to $80^{\circ}$ have been observed also. Analysis of droplet traces from consecutive images shows the influence of gravitational force on droplets with larger mass and smaller velocity values. Due to gravitation the resulting angular distribution of droplets became non-symmetric.

The high speed imaging of QSPA Kh-50 plasma interaction with the inclined tungsten target is presented in Fig. 2 For this case, the angular distribution of ejected droplets is also non-symmetric (Fig. 4). The maximal number of particles has been registered at angle $(35 \div 40)^{\circ}$ to the normal. Such particles flying towards the plasma impact, i.e., in upstream direction. However, quite large number of droplets turns to the downstream direction due to the influence of gravitational force and plasma stream pressure. It should be mentioned that the impacting energy density to the exposed surface depends on the plasma incidence angle to the target [8, 2]. A non-uniform distribution of the heat load along the target surface (for instance, due to formation of non-uniform shielding layer) can also cause a non-symmetric angular distribution of ejected droplets.

For heat load exceeding the melting threshold, the flying particles can be originated from melt surface due to Kelvin-Helmholtz or Rayleigh-Taylor instabilities [1]. Analysis of obtained experimental results and comparison with the results of numerical simulations [10, 9] allows conclusion that the generation of tungsten particles in the form of droplets may occur
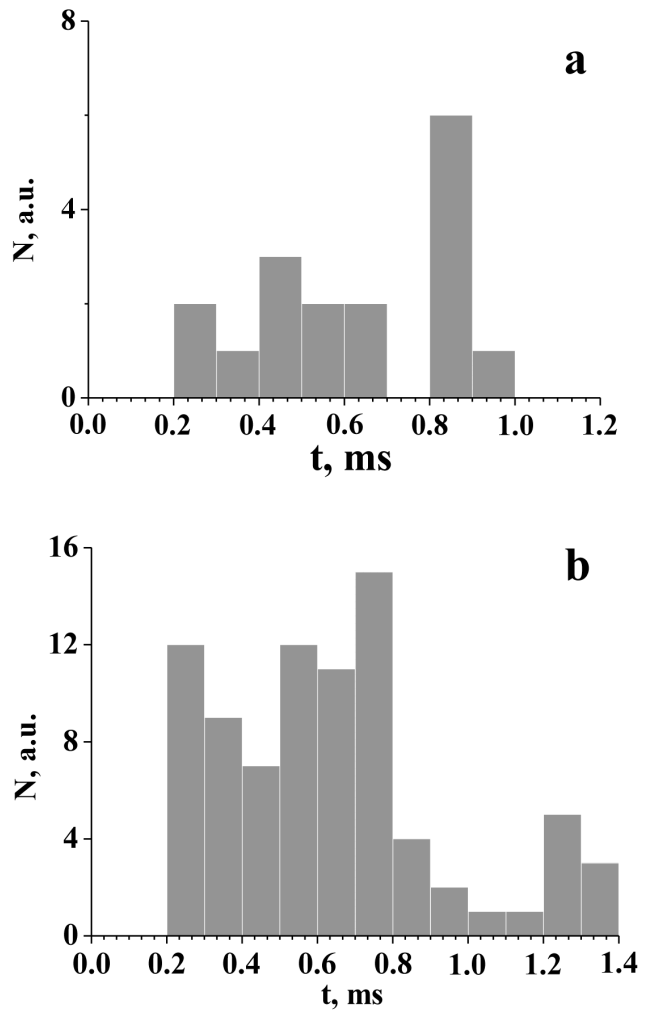

Figure 3. Amount of dust particles versus delay from start for different heat loads to target surface; a) $Q_{\text {surf }}=0.6 \mathrm{MJ} \mathrm{m}^{-2}$, b) $Q_{\text {surf }}=0.75 \mathrm{MJ} \mathrm{m}^{-2}$.

only during the plasma pulse and (as latest) few tens microseconds after the pulse end. Other particles may be exclusively solid dust that is generated due to elastic energy stored in stressed re-solidified tungsten surface layer. It is interesting to compare the results of QSPA Kh-50 plasma exposures with performed experiments on erosion product monitoring in QSPA-T facility 3, 1. Similar velocity of erosion products and the energy threshold of particles appearing have been observed. However, in our experiments with inclined irradiation the droplets are primarily ejected at small angles to the plasma jet. The reason for somewhat different results obtained in mentioned devices can be much larger plasma pressure and output electric currents in QSPA-T.

\section{Summary}

The results of erosion products monitoring from tungsten targets exposed to ITER ELM-like surface heat load at QSPA Kh-50 have been discussed. Plasma energy load to the target surfaces achieved $0.75 \mathrm{MJ} \mathrm{m}^{-2}$ and caused a pronounced surface melting.

The erosion products for tungsten targets have been registered with a high-speed digital camera both under normal and inclined plasma irradiation. Distributions of the particles in dependence on their start time from the target surface have been obtained for different heat loads. The number of particles grows significantly with increasing heat load. Erosion products have been observed with CCD camera only under 


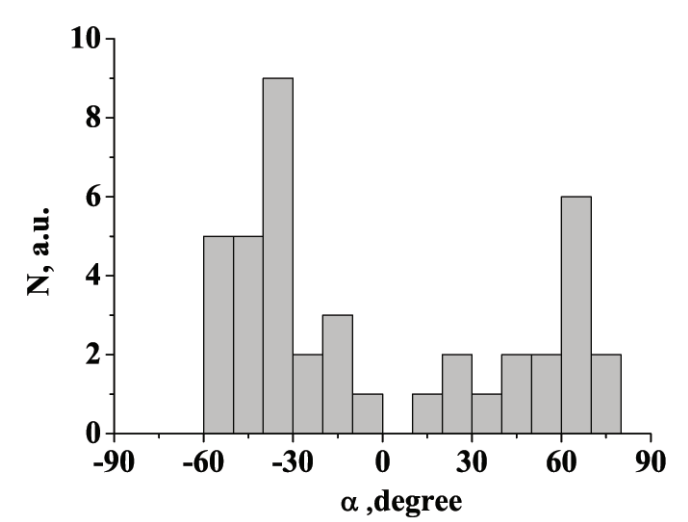

Figure 4. The angular distribution of ejected droplets for inclined $\left(35^{\circ}\right.$ to plasma jet) plasma irradiation.

heat load exceeding the cracking threshold. Maximal velocity of tungsten particles may achieve few tens of $\mathrm{m} / \mathrm{s}$. The main erosion mechanisms are found to be droplet ejection from the melt and solid dust origination during the brittle destruction of exposed tungsten surfaces due to the thermo-stress. For energy load below $0.75 \mathrm{MJ} \mathrm{m}^{-2}$, the start time of ejected particles from the surface is only weakly depended on the heat load value.

The measurement scheme applied in QSPA Kh-50 can be effectively used for investigations of dust dynamics in near-surface plasma in simulation studies of plasma interaction with ITER divertor surfaces.

\section{REFERENCES}

[1] B. Bazylev, et al. Experimental and theoretical investigation of droplet emission from tungsten melt layer. Fusion Engineering and Design 84:441, 2009.
[2] A. A. Chuvilo, et al. Calorimetric studies of the energy deposition on a material surface by plasma jets generated with qspa and mpc devices. NUKLEONIKA $\mathbf{5 7}: 49,2012$.

[3] I. E. Garkusha, et al. Experimental study of plasma energy transfer and material erosion under elm-like heat loads. J Nucl Mater 390/391:814, 2009.

[4] I. E. Garkusha, et al. Latest results from elm-simulation experiments in plasma accelerators. Phys Scr T135:014054, 2009.

[5] N. Klimov, et al. Experimental study of pfcs erosion and eroded material deposition under iter-like transient loads at the plasma gun facility qspa-t. Journal of Nuclear Materials 415:559, 2011.

[6] S. I. Krasheninnikov, R. D. Smirnov, D. L. Rudakov. Dust in magnetic fusion devices plasma phys. Control Fusion 53:083001, 2011.

[7] I. Landman, et al. Material surface damage under high pulse loads typical for elm bursts and disruptions in iter. Phys Scr 111:206, 2004.

[8] V. A. Makhlaj, et al. Simulation of iter edge localized modes' impacts on the divertor surfaces within plasma accelerators. Phys Scr T145:014061, 2011.

[9] S. Pestchanyi, et al. Estimation of the dust production rate from the tungsten armour after repetitive elm-like heat loads. PhysScr T145:014062, 2011.

[10] S. Pestchanyi, et al. Simulation of residual thermostress in tungsten after repetitive elm-like heat loads. Fusion Engineering and Design 86:1681, 2011.

[11] M. Rietha, et al. Review on the efda programme on tungsten materials technology and science. Journal of Nuclear Materials 417(1-3):463, 2011.

[12] V. I. Tereshin, et al. Powerful quasi-steady-state plasma accelerator for fusion experiments. Brazil $J$ Phys 32:165, 2002. 